\title{
Une généralisation d'une formule d'Erdelyi-Tricomi
}

\author{
François Batola
}

Résumé. On établit une relation intégrale généralisant un résultat d'ErdelyiTricomi. Pour cela on considère une solution de l'équation biconfluente de l'équation de Heun, dont on détermine l'intégrale de Fourier par le méthode des équations différentielles ordinaires [7], [9], [10].

La formule d'Erdelyi--Tricomi aparaît comme un cas particulier de notre résultat et s'obtient de façon naturelle en particularisant certains paramètres.

\section{Introduction}

On se propose d'établir dans ce travail une relation intégrale généralisant une formule d'Erdelyi-Tricomi [5], ([10], page 32 formule (4)). Ce problème a déjà été envisagé dans [1]; mais le point de vue adopté ici est différent.

Pour cela, on va utiliser l'équation différentielle ordinaire suivante:

$$
x y^{\prime \prime}+\left\{1+\alpha-\beta x-2 x^{2}\right\} y^{\prime}+\left\{(\gamma-2-\alpha) x-\frac{1}{2}[\delta+\beta(1+\alpha)]\right\} y=0 .
$$

Cette équation différentielle est connue comme étant l'équation biconfluente de l'équation de Heun [7] dont les propriétés ont été étudies d'abord dans [8] puis dans [2], [4], [3] et en détails dans [1].

Avant d'entrer dans le vif du sujet, rappelons brièvement quelques propriétés de l'équation biconfluente de l'équation de Heun dont nous aurons besoin dans ce travail.

\section{Quelques rappels et compléments}

1.1 Equation canonique. Généralement considérée comme l'équation canonique de la classe $\left(0,1,1_{4}\right)$, [7], [8], l'équation $(0.1)$ admet dans le voisinage de l'origine deux solutions linéairement indépendantes, lorsque $\alpha$ n'est pas un entier relatif:

$$
\begin{aligned}
& y_{1}(x)=N(\alpha, \beta, \gamma, \delta ; x) \\
& y_{2}(x)=x^{-\alpha} N(-\alpha, \beta, \gamma, \delta ; x) .
\end{aligned}
$$


La fonction $N(\alpha, \beta, \gamma, \delta ; x)$ est une fonction entière qu'il est commode de mettre sous la forme classique [1], [4], [2]:

$$
N(\alpha, \beta, \gamma, \delta ; x)=\sum_{v=0}^{\infty} \frac{A_{v}(\alpha, \beta, \gamma, \delta)}{(1+\alpha)_{v}} \frac{x^{v}}{v !}
$$

avec:

$$
\begin{array}{r}
A_{0}(\alpha, \beta, \gamma, \delta)=1 ; \quad A_{1}(\alpha, \beta, \gamma, \delta)=\frac{1}{2}[\delta+\beta(1+\alpha)] \\
A_{v+1}(\alpha, \beta, \gamma, \delta)=\left(v \beta+\frac{1}{2}[\delta+\beta(1+\alpha)]\right) A_{v}(\alpha, \beta, \gamma, \delta) \\
-v(v+\alpha)(\gamma-2-\alpha-2(v-1)) A_{v-1}(\alpha, \beta, \gamma, \delta) \quad v \geqq 1
\end{array}
$$

et avec:

$$
(\alpha)_{v}=\frac{\Gamma(\alpha+v)}{\Gamma(\alpha)}= \begin{cases}\alpha(\alpha+1) \ldots(\alpha+v-1) & v=1,2,3, \ldots \\ 1 & v=0\end{cases}
$$

Lorsquè $\alpha$ n'est pas un entier négatif, la fonction $N(\alpha, \beta, \gamma, \delta ; x)$ vérifie les identités survantes [8], [1], [4]:

$$
\begin{aligned}
& N(\alpha, \beta, \gamma, \delta ; x)=N(\alpha,-i \beta,-\gamma, i \delta ;-i x) e^{\beta x+x^{2}} \\
& N(\alpha, \beta, \gamma, \delta ; x)=N(\alpha,-\beta, \gamma,-\delta ;-x) .
\end{aligned}
$$

1.2 Comportement asymptotique. La fonction $N(\alpha, \beta, \gamma, \delta ; x)$ admet au voisinage de l'infini le comportement asymptotique suivant [8], [1]:

$$
N(\alpha, \beta, \gamma, \delta ; x) \sim k_{2}(\alpha, \beta, \gamma, \delta) e^{\beta x+x^{2}} x^{-\frac{1}{2}(\gamma+2+\alpha)} \quad x \rightarrow \infty
$$

où $k_{2}(\alpha, \beta, \gamma, \delta)$ représente une constante non élémentaire dépendant seulement des paramètres $\alpha, \beta, \gamma, \delta$. La détermination précise de cette constante est un problème difficile qui n'a pas été abordé dans [8], [1], [4]. On peut cependant donner le résultat suivant:

Proposition 1. La constante $k_{2}(\alpha, \beta, \gamma, \delta)$ qui intervient dans (1.6) est définie par la relation suivante:

$$
k_{2}(\alpha, \beta, \gamma, \delta)=\frac{\Gamma(1+\alpha)}{\Gamma\left(\frac{\alpha-\gamma}{2}\right) \Gamma\left(1+\frac{\alpha+\gamma}{2}\right)} J_{1+\frac{\alpha+\gamma}{2}}\left(\frac{\alpha+\gamma}{2}, \beta, \frac{3 \alpha-\gamma}{2}, \delta+\beta\left(\frac{\gamma-\alpha}{2}\right)\right)
$$

où $\Gamma(\alpha)$ désigne la fonction eulérienne Gamma et où

$$
J_{\lambda}(\alpha, \beta, \gamma, \delta)=\int_{0}^{\infty} x^{\lambda-1} e^{-\beta x-x^{2}} N(\alpha, \beta, \gamma, \delta ; x) d x
$$

intégrale absolument convergente pour $\operatorname{Re} \lambda>0$ et $\operatorname{Re} \frac{\gamma+\alpha}{2}-\lambda>-1$.

La constante $J_{\lambda}(\alpha, \beta, \gamma, \delta)$ peut être commodément exprimée à l'aide de l'intégrale de Faxen [6] souvent notée $F_{i}(\alpha, \lambda ; x)$ mais que nous noterons $\Gamma^{\alpha, \lambda}(x)$ 
pour bien rappeler la fonction eulérienne Gamma qu'elle généralise, et qui est définie par la relation:

$$
\Gamma^{\alpha, \lambda}(x)=\int_{0}^{\infty} e^{-\lambda t^{\alpha}-t} t^{x-1} d t \text { avec } \operatorname{Re} x>0
$$

A l'aide de cette intégrale et des coefficients $A_{k}(\alpha, \beta, \gamma, \delta)$ apparaissant dans (1.2) et définis par la relation de récurrence (1.3) la constante $J_{\lambda}(\alpha, \beta, \gamma, \delta)$ prend la forme:

$$
J_{\lambda}(\alpha, \beta, \gamma, \delta)=\frac{1}{2} \sum_{k=0}^{\infty} \frac{A_{k}(\alpha, \beta, \gamma, \delta)}{(1+\alpha)_{k} k !} \Gamma^{\frac{1}{2}, \beta}\left(\frac{\lambda+k}{2}\right) .
$$

On pose:

$$
(\alpha)_{e, k}^{\sigma, v}=\frac{\Gamma^{\sigma, v}(\alpha+\varrho k)}{\Gamma^{\sigma, v}(\alpha)}
$$

et on introduit la fonction non élémentaire:

$$
F_{Q}^{\sigma, v}(\alpha, \beta, \gamma, \delta ; \lambda ; c ; x)=\sum_{k=0}^{\infty} \frac{A_{k}(\alpha, \beta, \gamma, \delta)}{(c)_{k}}(\lambda)_{e, k}^{\sigma, v} \frac{x^{k}}{k !} .
$$

Cette fonction apparaît comme une généralisation de la fonction hypergéométrique de Gauss; de ce fait elle est liée à la fonction de Heun, solution de l'équation de Heun [7]. L'étude détaillée de la fonction (1.12) fera l'objet d'un travail ultérieur.

A l'aide de (1.12) l'expression de la constante $J_{\hat{\lambda}}(\alpha, \beta, \gamma, \delta)$ prend la forme simple:

$$
J_{\lambda}(\alpha, \beta, \gamma, \delta)=\frac{1}{2} \Gamma^{\frac{1}{2}, \beta}\left(\frac{\lambda}{2}\right) F_{\frac{1}{2}}^{\frac{1}{2}, \beta}\left(\alpha, \beta, \gamma, \delta ; \frac{\lambda}{2} ; 1+\alpha ; 1\right) .
$$

On peut donc énoncer le résultat suivant qui permet de préciser l'expression de la constante $k_{2}(\alpha, \beta, \gamma, \delta)$ :

Corollaire 1. La constante $k_{2}(\alpha, \beta, \gamma, \delta)$ qui intervient dans (1.6) est aussi définie par la relation suivante:

$$
\begin{gathered}
k_{2}(\alpha, \beta, \gamma, \delta)=\frac{1}{2} \frac{\Gamma(1+\alpha) \Gamma^{\frac{1}{2}, \beta}\left(\frac{1}{2}+\frac{\alpha+\gamma}{4}\right)}{\Gamma\left(\frac{\alpha-\gamma}{2}\right) \Gamma\left(1+\frac{\alpha+\gamma}{2}\right)} \\
\times F_{\frac{2}{2}}^{\frac{1}{2}, \beta}\left(\frac{\alpha+\gamma}{2}, \beta, \frac{3 \alpha-\gamma}{2}, \delta+\beta\left(\frac{\gamma-\alpha}{2}\right) ; \frac{1}{2}+\frac{\alpha+\gamma}{4} ; 1+\frac{\alpha+\gamma}{2} ; 1\right)
\end{gathered}
$$

où $\Gamma(\alpha)$ est la fonction eulérienne Gamma et où la fonction $F_{e}^{\sigma, v}$ est donnée par (1.12) et où la fonction $\Gamma^{\sigma, v}(x)$ est la fonction de Faxen définie par (1.9).

Le cas particulier de ces résultats sera examiné à la section 1.4 de ce paragraphe 1 . 
L'équation (0.1) admet aussi dans le voisinage de l'infini, entre autres, deux solutions linéairement indépendantes notées $B^{+}(\alpha, \beta, \gamma, \delta ; x)$ et $H^{+}(\alpha, \beta, \gamma, \delta ; x)$ dans le demi-plan de droite et deux autres solutions linéairement indépendantes notées $B^{-}(\alpha, \beta, \gamma, \delta ; x)$ et $H^{-}(\alpha, \beta, \gamma, \delta ; x)$ dans le demi-plan de gauche. Ces solutions possèdent le comportement asymptotique suivant [8], [1], [4]:

Au voisinage de l'infini

$$
\begin{array}{lll}
B^{+}(\alpha, \beta, \gamma, \delta ; x) \sim x^{\frac{1}{2}(\gamma-2-\alpha)} & x \rightarrow \infty \\
H^{+}(\alpha, \beta, \gamma, \delta ; x) \sim x^{-\frac{1}{2}(\gamma+2+\alpha)} e^{\beta x+x^{2}} & x \rightarrow \infty
\end{array}
$$

avec $-\frac{\pi}{2}+\varepsilon \leqq \arg x \leqq \frac{\pi}{2}-\varepsilon$

$$
\begin{array}{ll}
B^{-}(\alpha, \beta, \gamma, \delta ; x) \sim(-x)^{\frac{1}{2}(\gamma-2-\alpha)} & x \rightarrow \infty \\
H^{-}(\alpha, \beta, \gamma, \delta ; x) \sim(-x)^{-\frac{1}{2}(\gamma+2+\alpha)} e^{\beta x+x^{2}} & x \rightarrow \infty
\end{array}
$$

avec $\frac{\pi}{2}+\varepsilon \leqq \arg x \leqq 3 \frac{\pi}{2}-\varepsilon$.

Au voisinage de l'origine [1]

$$
\begin{gathered}
B^{+}(\alpha, \beta, \gamma, \delta ; x) \\
\sim \frac{2^{1+\frac{\alpha-\gamma}{2}} \Gamma(\alpha) 2^{-\alpha}}{\Gamma\left(1+\frac{\alpha-\gamma}{2}\right)} k_{2}\left(\frac{\alpha-\gamma}{2}, \beta,-\frac{3 \alpha+\gamma}{2}, \delta+\beta\left(\frac{\gamma+\alpha}{2}\right)\right) x^{-\alpha} \quad x \rightarrow 0
\end{gathered}
$$

et avec $\frac{\alpha-\gamma}{2}$ non entier négatif où la constante $k_{2}(\alpha, \beta, \gamma, \delta)$ est celle définie en (1.6), (1.7) ou (1.14)

$$
H^{+}(\alpha, \beta, \gamma, \delta ; x)=O\left(x^{-\alpha}\right) \quad x \rightarrow 0 .
$$

Nous n'explicitons pas plus ce dernier comportement car nous n'en aurons pas besoin.

Entre les fonctions $B^{+}(\alpha, \beta, \gamma, \delta ; x)$ et $B^{-}(\alpha, \beta, \gamma, \delta ; x)$ on a la relation de passage:

$$
B^{-}(\alpha, \beta, \gamma, \delta ; x)=B^{+}(\alpha,-\beta, \gamma,-\delta ;-x) \quad \operatorname{Re} x<0 .
$$

1.3 Equation transformée. Par la transformation élémentaire suivante:

$$
y(x)=e^{\beta x+x^{2}} u(x)
$$

l'équation canonique (0.1) se transforme en l'équation suivante:

$$
x u^{\prime \prime}+u^{\prime}\left\{1+\alpha+\beta x+2 x^{2}\right\}+u\left\{(\gamma+2+\alpha) x-\frac{1}{2}[\delta-\beta(1+\alpha)]\right\}=0
$$


avec la solution

$$
u(x)=e^{-\beta x-x^{2}} y(\alpha, \beta, \gamma, \delta ; x)
$$

où $y(\alpha, \beta, \gamma, \delta ; x)$ désigne une solution de l'équation canonique $(0.1)$.

De même, par la transformation:

$$
y(x)=x^{-\alpha} e^{\beta x+x^{2}} u(x)
$$

l'équation canonique (0.1) se transforme en l'équation:

$$
x u^{\prime \prime}+u^{\prime}\left\{1-\alpha+\beta x+2 x^{2}\right\}+u\left\{(\gamma+2-\alpha) x-\frac{1}{2}[\delta-\beta(1-\alpha)]\right\}=0
$$

avec la solution suivante:

$$
u(x)=x^{\alpha} e^{-\beta x-x^{2}} y(\alpha, \beta, \gamma, \delta ; x)
$$

où $y(\alpha, \beta, \gamma, \delta ; x)$ désigne une solution de l'équation canonique $(0.1)$.

1.4 Cas particuliers. Dans le cas particulier où $\beta=\delta=0$, on a [8], [1], [2], [4]:

$$
N(\alpha, 0, \gamma, 0 ; x)=\Phi\left(\frac{\alpha+2-\gamma}{4}, 1+\frac{\alpha}{2} ; x^{2}\right)
$$

où $\Phi(a, c ; x)$ est la fonction confluente de Kummer souvent notée aussi ${ }_{1} F_{1}(a, c ; x)$.

L'équation bien connue de Kummer [9], [10] est un cas particulier de (0.1).

On a aussi le résultat suivant:

$$
B^{+}(\alpha, 0, \gamma, 0 ; x)=\Psi\left(\frac{\alpha+2-\gamma}{4}, 1+\frac{\alpha}{2} ; x^{2}\right) \quad \operatorname{Re} x>0
$$

où $\Psi(a, c ; x)$ est la fonction de Tricomi ([10], page 17).

L'importance de la fonction $F_{Q}^{\sigma, \nu}$ introduite en (1.12) peut être bien comprise si on examine un cas particulier donné par la proposition suivante:

\section{Proposition 2.}

$$
F_{\frac{1}{2}}^{\sigma, 0}(\alpha, 0, \gamma, 0 ; \lambda ; 1+\alpha ; x)={ }_{2} F_{1}\left(\frac{\alpha+2-\gamma}{4}, \lambda ; 1+\frac{\alpha}{2} ; x^{2}\right)
$$

où ${ }_{2} F_{1}(a, b, c ; x)$ est la fonction hypergéométrique de Gauss.

Preuve. Considérons (1.12) et prenons $\beta=\delta=0$. On a :

$$
F_{\varrho}^{\sigma, v}(\alpha, 0, \gamma, 0 ; \lambda ; 1+\alpha ; x)=\sum_{k=0}^{\infty} \frac{A_{k}(\alpha, 0, \gamma, 0)}{(1+\alpha)_{k}}(\lambda)_{\varrho, k}^{\sigma, \gamma} \frac{x^{k}}{k !} .
$$

Or

$$
\begin{aligned}
& A_{2 k+1}(\alpha, 0, \gamma, 0)=0 \\
& A_{2 k}(\alpha, 0, \gamma, 0)=(-1)^{k} 1 \cdot 3 \cdot 5 \ldots(2 k-1)(1+\alpha)(3+\alpha)(5+\alpha) \ldots(2 k-1+\alpha) . \\
& \quad \theta(\theta-4)(\theta-8) \ldots(\theta-4(k-1)) \text { avec } \theta=\gamma-2-\alpha .
\end{aligned}
$$


On a donc

$$
F_{\varrho}^{\sigma, v}(\alpha, 0, \gamma, 0 ; \lambda ; 1+\alpha ; x)=\sum_{k=0}^{\infty} \frac{A_{2 k}(\alpha, 0, \gamma, 0)}{(1+\alpha)_{2 k}}(\lambda)_{e, 2 k}^{\sigma, v} \frac{x^{2 k}}{(2 k) !} .
$$

Et comme $(1+\alpha)_{2 k}=2^{2 k}\left(\frac{1+\alpha}{2}\right)_{k}\left(1+\frac{\alpha}{2}\right)_{k}$ on a donc

$$
\begin{gathered}
F_{Q}^{\sigma, v}(\alpha, 0, \gamma, 0 ; \lambda ; 1+\alpha ; x) \\
=\sum_{k=0}^{\infty} \frac{(-1)^{k} 1 \cdot 3 \cdot 5 \ldots(2 k-1) \theta(\theta-4)(\theta-8) \ldots(\theta-4(k-1))(\lambda)_{Q, 2 k}^{a, v}}{2^{k}\left(1+\frac{\alpha}{2}\right)_{k}} \frac{x^{2 k}}{(2 k) !}
\end{gathered}
$$

ou encore

$$
=\sum_{k=0}^{\infty} \frac{\left(-\frac{\theta}{4}\right)\left(-\frac{\theta}{4}+1\right)\left(-\frac{\theta}{4}+2\right) \ldots\left(-\frac{\theta}{4}+k-1\right)(\lambda)_{e, v k}^{\sigma, v}}{\left(1+\frac{\alpha}{2}\right)_{k}^{\sigma, v}(\alpha, 0, \gamma, 0 ; \lambda ; 1+\alpha ; x)} \frac{x^{2 k}}{k !} .
$$

Il en résulte:

$$
F_{\frac{1}{2}}^{\sigma, 0}(\alpha, 0, \gamma, 0 ; \lambda ; 1+\alpha ; x)=\sum_{k=0}^{\infty} \frac{\left(-\frac{\theta}{4}\right)_{k}(\lambda)_{k}}{\left(1+\frac{\alpha}{2}\right)_{k}} \frac{\left(x^{2}\right)^{k}}{k !}
$$

Et le résultat cherché en découle puisque $\theta=\gamma-2-\alpha$. Q.E.D.

\section{Corollaire 2.}

$$
\begin{gathered}
J_{\lambda}(\alpha, 0, \gamma, 0)=\frac{1}{2} \frac{\Gamma\left(\frac{\lambda}{2}\right) \Gamma\left(1+\frac{\alpha}{2}\right) \Gamma\left(\frac{2+\alpha+\gamma}{4}-\frac{\lambda}{2}\right)}{\Gamma\left(\frac{\alpha+2+\gamma}{4}\right) \Gamma\left(1+\frac{\alpha}{2}-\frac{\lambda}{2}\right)}, \\
k_{2}(\alpha, 0, \gamma, 0)=\frac{\Gamma\left(1+\frac{\alpha}{2}\right)}{\Gamma\left(\frac{\alpha+2-\gamma}{4}\right)} .
\end{gathered}
$$

Preuve. D’après une propriété bien connue de la fonction hypergéométrique de Gauss on a:

$$
{ }_{2} F_{1}(a, b, c ; 1)=\frac{\Gamma(c) \Gamma(c-b-a)}{\Gamma(c-a) \Gamma(c-b)} .
$$


11 en résulte:

$$
\begin{gathered}
F_{\frac{1}{2}}^{\sigma, 0}(\alpha, 0, \gamma, 0 ; \lambda ; 1+\alpha ; 1)={ }_{2} F_{1}\left(\frac{\alpha+2-\gamma}{4}, \lambda, 1+\frac{\alpha}{2} ; 1\right) \\
=\frac{\Gamma\left(1+\frac{\alpha}{2}\right) \Gamma\left(\frac{\alpha+\gamma+2}{4}-\lambda\right)}{\Gamma\left(\frac{\alpha+2+\gamma}{4}\right) \Gamma\left(1+\frac{\alpha}{2}-\lambda\right)} .
\end{gathered}
$$

En reportant ce résultat dans (1.13) on a:

$$
J_{\lambda}(\alpha, 0, \gamma, 0)=\frac{1}{2} \frac{\Gamma\left(\frac{\lambda}{2}\right) \Gamma\left(1+\frac{\alpha}{2}\right) \Gamma\left(\frac{\alpha+2+\gamma}{4}-\frac{\lambda}{2}\right)}{\Gamma\left(\frac{\alpha+2+\gamma}{4}\right) \Gamma\left(1+\frac{\alpha}{2}-\frac{\lambda}{2}\right)}
$$

d'oú

$$
J_{1+\frac{\alpha+\gamma}{2}}\left(\frac{\alpha+\gamma}{2}, 0, \frac{\alpha-\gamma}{2}, 0\right)=\frac{1}{2 \sqrt{\pi}} \frac{\Gamma\left(\frac{\alpha+2+\gamma}{4}\right) \Gamma\left(1+\frac{\alpha+\gamma}{4}\right) \Gamma\left(\frac{\alpha-\gamma}{4}\right)}{\Gamma\left(\frac{\alpha+1}{2}\right)} .
$$

En reportant d'abord ce résultat dans (1.7), puis en transformant $\Gamma(1+\alpha), \Gamma\left(\frac{\alpha-\gamma}{2}\right)$, $\Gamma\left(1+\frac{\alpha+\gamma}{2}\right)$ à l'aide de la formule de duplication et en simplifiant enfin le résultat obtenu, on obtient le résultat annoncé. Q.E.D.

\section{Une relation intégrale préliminaire}

Considérons l'intégrale de Fourier donnée par:

$$
S(z)=\int_{-\infty}^{\infty} e^{i z x} u(x) d x \quad z \in \mathbf{R}
$$

avec

$$
u(x)=e^{-\beta x-x^{2}} N(\alpha, \beta, \gamma, \delta ; x)
$$

dont le comportement asymptotique au voisinage de l'infini est donné par:

$$
u(x)=O\left(x^{-\frac{1}{2}(\gamma+2+\alpha)}\right) \quad|x| \rightarrow \infty .
$$

Donc l'intégrale de Fourier (2.1) converge absolument si

$$
\operatorname{Re}(\gamma+\alpha)>0 \text { et si } z \in \mathbf{R} .
$$


L'équation différentielle ordinaire vérifiée par $u(x)$ est celle donnée en (1.21) et que l'on ré-écrit:

$$
x u^{\prime \prime}+u^{\prime}\left\{1+\alpha+\beta x+2 x^{2}\right\}+u\left\{(\gamma+2+\alpha) x-\frac{1}{2}[\delta-\beta(1+\alpha)]\right\}=0 .
$$

Observons que les intégrales de Fourier des fonctions suivantes:

$$
u(x) ; \quad x u(x) ; \quad u^{\prime}(x) ; \quad x u^{\prime}(x) ; \quad x^{2} u^{\prime}(x) ; \quad x u^{\prime \prime}(x)
$$

qui entrent dans l'expression de l'équation (2.4) convergent absolument si $\operatorname{Re}(\gamma+\alpha)>2$ et si $z \in \mathbf{R}$. En prenant la transformée de Fourier de (2.4) selon la formule (2.1) on a l'équation suivante:

$$
2 z S^{\prime \prime}+S^{\prime}\left\{z^{2}+i \beta z-(\gamma-2+\alpha)\right\}+S\left\{z(1-\alpha)+\frac{1}{2}[i \delta+i \beta(1-\alpha)]\right\}=0
$$

En posant $z=2 w$ on a:

$$
w S^{\prime \prime}+S^{\prime}\left\{2 w^{2}+i \beta w+1-\frac{\gamma+\alpha}{2}\right\}+\left\{2 w(1-\alpha)+\frac{1}{2}[i \delta+i \beta(1-\alpha)]\right\} S=0 .
$$

C'est une équation du type:

$$
w u^{\prime \prime}+u^{\prime}\left\{1-\tilde{\alpha}+\tilde{\beta} w+2 w^{2}\right\}+u\left\{(\tilde{\gamma}+2-\tilde{\alpha}) w-\frac{1}{2}[\tilde{\delta}-\tilde{\beta}(1-\tilde{\alpha})]\right\}=0
$$

déjà rencontrée en (1.24) avec:

$$
\tilde{\alpha}=\frac{\gamma+\alpha}{2} ; \tilde{\beta}=i \beta ; \tilde{\gamma}=\frac{\gamma-3 \alpha}{2} ; \tilde{\delta}=-i\left[\delta+\beta\left(\frac{\gamma-\alpha}{2}\right)\right] .
$$

Donc l'équation différentielle ordinaire (2.7) admet la solution suivante:

$$
S(z)=\left(\frac{z}{2}\right)^{\tilde{\alpha}} e^{-\tilde{\beta} \frac{z}{2}-\frac{z^{2}}{4}} y\left(\tilde{\alpha}, \tilde{\beta}, \tilde{\gamma}, \tilde{\delta} ; \frac{z}{2}\right)
$$

où $y(\tilde{\alpha}, \tilde{\beta}, \tilde{\gamma}, \tilde{\delta} ; z)$ est une solution de l'équation canonique $(0.1)$ avec des paramètres appropriés. Grâce à (2.1) et (2.10) on a donc obtenu le résultat préliminaire suivant:

$$
\left(\frac{z}{2}\right)^{\tilde{\alpha}} e^{-\tilde{\beta} \frac{z}{2} z^{2}} y\left(\tilde{\alpha}, \tilde{\beta}, \tilde{\gamma}, \tilde{\delta} ; \frac{z}{2}\right)=\int_{-\infty}^{\infty} e^{i z x} e^{-\beta x-x^{2}} N(\alpha, \beta, \gamma, \delta ; x) d x \quad z \in \mathbf{R}
$$

Cette relation nous sera d'une très grande utilité dans la suite de ce travail. 


\section{Notre résultat principal}

On va distinguer 2 cas:

3.1 Premier cas: $z$ réel positif. Les fonctions $B^{+}(\alpha, \beta, \gamma, \delta ; z)$ et $H^{+}(\alpha, \beta, \gamma, \delta ; z)$ constituent un système fondamental de l'équation canonique $(0.1)$ dans le demiplan de droite. On peut donc écrire la fonction $y(\tilde{\alpha}, \tilde{\beta}, \tilde{\gamma}, \tilde{\delta} ; z)$ de $(2.11)$ sous la forme:

$$
y(\tilde{\alpha}, \tilde{\beta}, \tilde{\gamma}, \tilde{\delta} ; z)=\tau_{1} B^{+}(\tilde{\alpha}, \tilde{\beta}, \tilde{\gamma}, \tilde{\delta} ; z)+\tau_{2} H^{+}(\tilde{\alpha}, \tilde{\beta}, \tilde{\gamma}, \tilde{\delta} ; z)
$$

où $\tau_{1}$ et $\tau_{2}$ sont deux constantes dépendant seulement des paramètres $\tilde{\alpha}, \tilde{\beta}, \tilde{\gamma}, \tilde{\delta}$.

Dans ces conditions la relation (2.11) s'écrit:

$$
\begin{gathered}
\left(\frac{z}{2}\right)^{\tilde{\alpha}} e^{-\tilde{\beta} \frac{z}{2}-\frac{z^{2}}{4}}\left(\tau_{1} B^{+}\left(\tilde{\alpha}, \tilde{\beta}, \tilde{\gamma}, \tilde{\delta} ; \frac{z}{2}\right)+\tau_{2} H^{+}\left(\tilde{\alpha}, \tilde{\beta}, \tilde{\gamma}, \tilde{\delta} ; \frac{z}{2}\right)\right) \\
=\int_{-\infty}^{\infty} e^{i z x} e^{-\beta x-x^{2}} N(\alpha, \beta, \gamma, \delta ; x) d x
\end{gathered}
$$

Pour déterminer les constantes $\tau_{1}$ et $\tau_{2}$ on va considérer séparément le comportement asymptotique des deux membres de (3.2): au voisinage de l'infini de ler membre de (3.2) se comporte comme suit:

$$
\tau_{1}\left(\frac{z}{2}\right)^{\frac{1}{2}(\gamma-\alpha-2)} e^{-i \beta \frac{z}{2}-\frac{z^{2}}{4}}+\tau_{2}\left(\frac{z}{2}\right)^{\alpha-1} \quad z \rightarrow \infty
$$

Il y a deux cas à distinguer; le cas $\operatorname{Re} \alpha>1$ et le cas $\operatorname{Re} \alpha \leqq 1$.

Dans ce travail on va considérer seulement le cas $\operatorname{Re} \alpha>1$ l'autre cas étant réservé pour une étude ultérieure.

Ainsi, dans le cas où $\operatorname{Re} \alpha>1$, le terme prépondérant dans (3.3) lorsque $z \rightarrow \infty$ est $\tau_{2}\left(\frac{z}{2}\right)^{\alpha-1}$. Et comme le deuxième membre de (3.2) est $o(1)$ lorsque $|z| \rightarrow \infty$ (d'après le théorème de Riemann-Lebesgue) on en déduit que:

$$
\tau_{2}=0
$$

Et donc (3.2) devient:

$$
\tau_{1}\left(\frac{z}{2}\right)^{\tilde{\alpha}} e^{-\tilde{\beta} \frac{z}{2}-\frac{z^{2}}{4}} B^{+}\left(\tilde{\alpha}, \tilde{\beta}, \tilde{\gamma}, \tilde{\delta} ; \frac{z}{2}\right)=\int_{-\infty}^{\infty} e^{i z x} e^{-\beta x-x^{2}} N(\alpha, \beta, \gamma, \delta ; x) d x .
$$

Considérons maintenant cette expression au voisinage de zéro. On se reportera à (1.17) pour le comportement de la fonction $B+(\alpha, \beta, \gamma, \delta ; x)$ au voisinage de 
l'origine. En comparant le comportement asymptotique des deux membres de (3.5) on a:

$$
\tau_{1}=\frac{J_{1}(\alpha, \beta, \gamma, \delta) \Gamma\left(1+\frac{\tilde{\alpha}-\tilde{\gamma}}{2}\right)}{\Gamma(\tilde{\alpha}) 2^{1-\frac{\tilde{\alpha}+\tilde{\beta}}{2}} k_{2}\left(\frac{\tilde{\alpha}-\tilde{\gamma}}{2}, \tilde{\beta},-\frac{\tilde{\alpha}+\tilde{\gamma}}{2}, \tilde{\delta}+\tilde{\beta}\left(\frac{\tilde{\gamma}+\tilde{\alpha}}{2}\right)\right)}
$$

ou encore

$$
\tau_{1}(\tilde{\alpha}, \tilde{\beta}, \tilde{\gamma}, \tilde{\delta})=\frac{J_{1}(\alpha, \beta, \gamma, \delta) \Gamma\left(1-\frac{\tilde{\alpha}+\tilde{\gamma}}{2}\right)}{2^{1-\frac{\tilde{\alpha}+\tilde{\gamma}}{2}} J_{1-\frac{\tilde{\alpha}+\tilde{\gamma}}{2}}\left(-\frac{\tilde{\alpha}+\tilde{\gamma}}{2}, \tilde{\beta}, \frac{3 \tilde{\alpha}-\tilde{\gamma}}{2}, \tilde{\delta}+\tilde{\beta}\left(\frac{\tilde{\gamma}-\tilde{\alpha}}{2}\right)\right)} .
$$

On a ainsi obtenu le résultat fondamental suivant:

Théorème 1. Entre la fonction $B^{+}(\alpha, \beta, \gamma, \delta ; z)$ et la fonction $N(\alpha, \beta, \gamma, \delta ; z)$ on a la relation intégrale suivante:

$$
\begin{gathered}
\tau_{1}(\tilde{\alpha}, \tilde{\beta}, \tilde{\gamma}, \tilde{\delta})\left(\frac{z}{2}\right)^{\tilde{\alpha}} e^{-\tilde{\beta} \frac{z}{2}-\frac{z^{2}}{4}} B^{+}\left(\tilde{\alpha}, \tilde{\beta}, \tilde{\gamma}, \tilde{\delta} ; \frac{z}{2}\right) \\
\quad=\int_{-\infty}^{\infty} e^{i z x} N(\alpha,-i \beta,-\gamma, i \delta ;-i x) d x
\end{gathered}
$$

$z>0 ; \operatorname{Re} \alpha>1 ; \operatorname{Re} \frac{\gamma+\alpha}{2}>1 ; \tau_{1}(\tilde{\alpha}, \tilde{\beta}, \tilde{\gamma}, \tilde{\delta})$ donnée par (3.6) ou (3.7) et $\tilde{\alpha}, \tilde{\beta}, \tilde{\gamma}, \tilde{\delta}$ étant définis par (2.9).

Ceci est notre résultat principal qui généralise le résultat d'Erdelyi-Tricomi [5], [10]. Un cas particulier en sera donna au 4.

3.2 Deuxième cas: $z$ réel négatif. Comme dans le cas précédent on aura le résultat suivant:

Corollaire 3. Entre la fonction $B^{+}(\alpha, \beta, \gamma, \delta ; z)$ et la fonction $N(\alpha, \beta, \gamma, \delta ; z)$ on a aussi la relation intégrale suivante:

$$
\begin{gathered}
\tau_{1}(\tilde{\alpha},-\tilde{\beta}, \tilde{\gamma},-\tilde{\delta})\left(-\frac{z}{2}\right)^{\tilde{\alpha}} e^{-\tilde{\beta} \frac{z}{2}-\frac{z^{2}}{4}} B^{+}\left(\tilde{\alpha},-\tilde{\beta}, \tilde{\gamma},-\tilde{\delta} ;-\frac{z}{2}\right) \\
=\int_{-\infty}^{\infty} e^{i z x} N(\alpha,-i \beta,-\gamma, i \delta ;-i x) d x
\end{gathered}
$$

$z<0 ; \operatorname{Re} \alpha>1 ; \operatorname{Re} \frac{\gamma+\alpha}{2}>1 ; \tau_{1}(\tilde{\alpha}, \tilde{\beta}, \tilde{\gamma}, \tilde{\delta})$ donnée par (3.6) ou (3.7) et $\tilde{\alpha}, \tilde{\beta}, \tilde{\gamma}, \tilde{\delta}$ étant définis par (2.9). 
Preuve. Dans la relation (3.8) du théorème 1, changer d'abord $z$ en $-z$ puis dans l'intégrale changer $x$ et $-x$. Ensuite changer dans les deux membres $\beta$ en $-\beta$ et $\delta$ en $-\delta$. On applique ensuite les relations (1.5) au second membre. Et le résultat cherché en découle immédiatement. Q.E.D.

\section{Les cas particuliers importants}

On a d'abord besoin du lemme suivant:

\section{Lemme 1.}

$$
\tau_{1}(\tilde{\alpha}, 0, \tilde{\gamma}, 0)=\sqrt{\pi} \frac{\Gamma\left(1+\frac{\alpha}{2}\right)}{\Gamma\left(\frac{\alpha+2+\gamma}{4}\right)}
$$

avec $\tilde{\alpha}, \tilde{\gamma}$ donnés par (2.9).

Preuve. Considérons l'expression (3.7) et prenons $\beta=\delta=0$; on a:

$$
\tau_{1}(\tilde{\alpha}, 0, \tilde{\gamma}, 0)=\frac{J_{1}(\alpha, 0, \gamma, 0) \Gamma\left(1-\frac{\tilde{\alpha}+\tilde{\gamma}}{2}\right)}{2^{1-\frac{\tilde{\alpha}+\tilde{\gamma}}{2}} J_{1-\frac{\tilde{\alpha}+\tilde{\gamma}}{2}}\left(-\frac{\tilde{\alpha}+\tilde{\gamma}}{2}, 0, \frac{3 \tilde{\alpha}-\tilde{\gamma}}{2}, 0\right)} .
$$

On utilise ensuite (1.30) du corollaire 2 ainsi que la formule bien connue de duplication. On utilise aussi (2.9). Après simplification le résultat cherché en découle immédiatement. Q.E.D.

On peut énoncer maintenant le résultat important suivant:

Théorème 2. Entre la fonction $\Psi(a, c ; x)$ de Tricomi et la fonction confluente de Kummer $\Phi(a, c ; x)$ on a la relation intégrale:

(4.2) $\frac{\sqrt{\pi}}{2} \frac{1}{\Gamma(a)}\left(\frac{z}{2}\right)^{2 a-1} e^{-\frac{z^{2}}{4}} \Psi\left(c-\frac{1}{2}, a+\frac{1}{2} ; \frac{z^{2}}{4}\right)=\int_{0}^{\infty} \cos z x \Phi^{*}\left(a, c ;-x^{2}\right) d x$

avec

$$
z>0 ; \operatorname{Re} c>\frac{3}{2} ; \operatorname{Re} a>1 ; \Phi^{*}(a, c ; x)=\frac{1}{\Gamma(c)} \Phi(a, c ; x)
$$


Preuve. Considérons la relation (3.8) de notre théorème 1. Prenons $\beta=\delta=0$ et donc $\tilde{\beta}=\tilde{\delta}=0$. Alors en utilisant $(1.26)$ et (1.27) on a:

$$
\begin{gathered}
\tau_{1}(\tilde{\alpha}, 0, \tilde{\gamma}, 0)\left(\frac{z}{2}\right)^{\tilde{\alpha}} e^{-\frac{z^{2}}{2}} \Psi\left(\frac{\tilde{\alpha}+2-\tilde{\gamma}}{4}, 1+\frac{\tilde{\alpha}}{2} ; \frac{z^{2}}{4}\right) \\
=\int_{-\infty}^{\infty} e^{i z x} \Phi\left(\frac{\alpha+2+\gamma}{4}, 1+\frac{\alpha}{2} ;-x^{2}\right) d x \\
z>0 ; \operatorname{Re} \alpha>1 ; \operatorname{Re} \frac{\gamma+\alpha}{2}>1 .
\end{gathered}
$$

Il en résulte:

$$
\begin{gathered}
\frac{1}{2} \tau_{1}(\tilde{\alpha}, 0, \tilde{\gamma}, 0)\left(\frac{z}{2}\right)^{\tilde{\alpha}} e^{-\frac{z^{2}}{4}} \Psi\left(\frac{\tilde{\alpha}+2-\tilde{\gamma}}{4}, 1+\frac{\tilde{\alpha}}{2} ; \frac{z^{2}}{4}\right) \\
=\int_{0}^{\infty} \cos z x \Phi\left(\frac{\alpha+2+\gamma}{4}, 1+\frac{\alpha}{2} ;-x^{2}\right) d x \\
z>0 ; \operatorname{Re} \alpha>1 ; \operatorname{Re} \frac{\gamma+\alpha}{2}>1 .
\end{gathered}
$$

On pose:

$$
\frac{\alpha+2+\gamma}{4}=a ; 1+\frac{\alpha}{2}=c
$$

On a donc:

$$
\tilde{\alpha}=\frac{\alpha+\gamma}{2}=2 a-1 ; \frac{\tilde{\alpha}+2-\tilde{\gamma}}{4}=c-\frac{1}{2} ; 1+\frac{\tilde{\alpha}}{2}=a+\frac{1}{2} .
$$

En reportant (4.4), (4.5) et (4.1) dans (4.3) et en se rappelant que $\Phi^{*}(a, c ; x)=$ $\frac{1}{\Gamma(c)} \Phi(a, c ; x)$ on obtient le résultat cherché. Q.E.D.

On a ainsi retrouvé un résultat d'Erdelyi-Tricomi [5], ([10] page 32 formule (4)).

A partir de notre Corollaire 3 on aura le résultat suivant:

Corollaire 4. Entre la fonction $\Psi(a, c ; x)$ et la fonction $\Phi(a, c ; x)$ on a aussi la relation intégrale suivante:

$$
\begin{gathered}
\frac{\sqrt{\pi}}{2} \frac{1}{\Gamma(a)}\left(-\frac{z}{2}\right)^{2 a-1} e^{-\frac{z^{2}}{4}} \Psi\left(c-\frac{1}{2}, a+\frac{1}{2} ; \frac{z^{2}}{4}\right) \\
=\int_{0}^{\infty} \cos z x \Phi^{*}\left(a, c ;-x^{2}\right) d x \\
z<0 ; \operatorname{Re} c>\frac{3}{2} ; \operatorname{Re} a>1 ; \Phi^{*}(a, c ; x)=\frac{1}{\Gamma(c)} \Phi(a, c ; x) .
\end{gathered}
$$

Ce résultat ne figure pas dans [10], mais peut être facilement retrouvé à partir de (4.2). 


\section{Bibliographie}

1. Batola, F., Quelques propriétés de l'équation biconfluente de l'équation de Heun. Thèse de 3ème cycle. Université Pierre et Marie Curie. Paris VI (1977).

2. Batola, F., Sur une généralisation des polynômes d'Hermite. Annales de la Société Scientifique de Bruxelles. 93 I, (1979), 17-38; MR 80i : 33018.

3. Batola, F., Sur l'équation de Schrödinger à potentiel singulier de Heun, à paraître.

4. Decarreau, A., Maroni, P. and Robert, A., Sur les équations confluentes de l'équation de Heun, Annales de la Société Scientifique de Bruxelles. 92 III, (1978), 151-189.

5. ERdelyI, A., Gewisse Reihentransformationen die mit der linearen Transformationsformel der Thetafunktion zusammenhängen. Compositio Math. 4 (1937), $406-423$.

6. FAXEN, H., Expansion in series of the integral $\int_{y}^{x} \exp \left[-x\left(t \pm t^{-n}\right)\right] t^{2} d t$, Ark. Math. Astr. Fys. 15 (1921), 1-57.

7. InCE, E. L., Ordinary differential equations, Dover Publications, Inc, (1956) New York.

8. Maroni, P., Sur la forme biconfluente de l'équation de Heun, C.R. Acad. Sc. Paris 264A (1967), 503-505.

9. Tricomi, F. G., Differential Equations, Blackie and Sons limited reprint (1966) London, Bombay.

10. Tricomi, F. G., Fonctions hypergéométriques confluentes, Mémorial des Sciences Mathématiques, fasc CXL, Gauthier-Villars, Paris, 1960. 